\section{Comments on Panksepp et al}

\author{
CHARLES R. GALLISTEL, University of \\ Pennsylvania, Philadelphia, Pa. 19104
}

In their replication of Gallistel (1967), Panksepp, Gandelman, \& Trowill (1968) regret the absence of trial-by-trial and day-by-day data in our report. We now give those data in Figs. 1 and 2.

As regards the differing interpretations, it is not clear that there is any but a terminological difference at the level of these studies. We concluded that the data "eliminated the possibility that the unusual effect of intertrial interval on [ESB] rewarded performance results from a change in the incentive value of [ESB] : The effect appears after the change in interval but before the rat has received a reward under the new conditions." We do not think there is any disagreement about this: an effect cannot precede its cause. We ascribed the effect to a drive-inducing property of ESB, defining drive as "whatever produces changes in the direction and avidity of behavior in the absence of changes in the animal's knowledge of the reward contingencies [size of the reward] ."Pankseppet al ascribe the effect to an incentive-drive interaction that produces a temporary increase in arousal. Thus, in their terminology, a shifting arousal causes changes in the avidity (at least) of responses in the absence of a change in the animal's knowledge of the reward contingencies; whereas in our terminology shifting drive level causes these changes.

One nonterminological difference in the two formulations might be that in calling the effect an arousal effect they mean to imply that it has no directive properties. Our interpretation implies that the effect has directive properties as well as energizing properties. Since these were not choice studies, the data do not bear on this question. We attribute directive properties to the effect because Deutsch, Adams, \& Metzner (1964) have shown that the more recently a thirsty rat has been primed with ESB the more likely it is to choose an ESB reward in preference to a water reward.

Another nonterminological difference is that their formulation implies that at some more molecular level the drive effect (or arousal effect) is a secondary consequence of activating a reinforcement system, whereas we imply that the effect arises from direct activation of another system. Again, the data here provide no evidence for deciding this. The only direct evidence comes from the Deutsch (1964) study showing that the refractory period of the drive (or arousal) system is not the same as were being activated indirectly through the other system.

Panksepp et al emphasize the regression to the baseline in their data. The effect seems minimal and its implications unclear. How can a regression effect support one hypothesis over the other when at this level the two hypotheses differ only in terminology? How does their hypothesis explain the regression of the $60-5$ group (three of their five regressing Ss were in this group)? In other words, what change in expectancy makes the 60-5 group slow down as postshift testing progresses? And, more importantly, the refractory period of the reward system. (We have replicated this study in our lab, using a runway. 1 ) It is likely that the refractory periods in question are neural refractory periods, but, even if they are not one would not obtain two different refractory period values if the one system

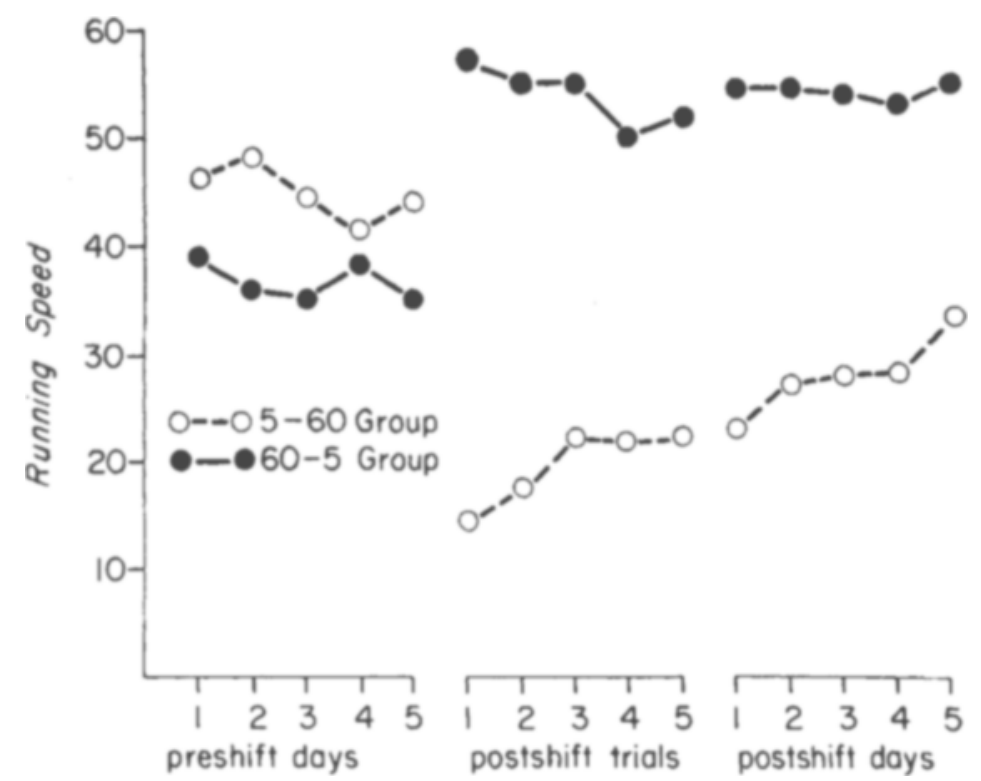

Fig. 1. Group mean performances on the last five preshift days (20 trials/day), the first five postshift trials, and the five postshift days ( 20 trials/day).

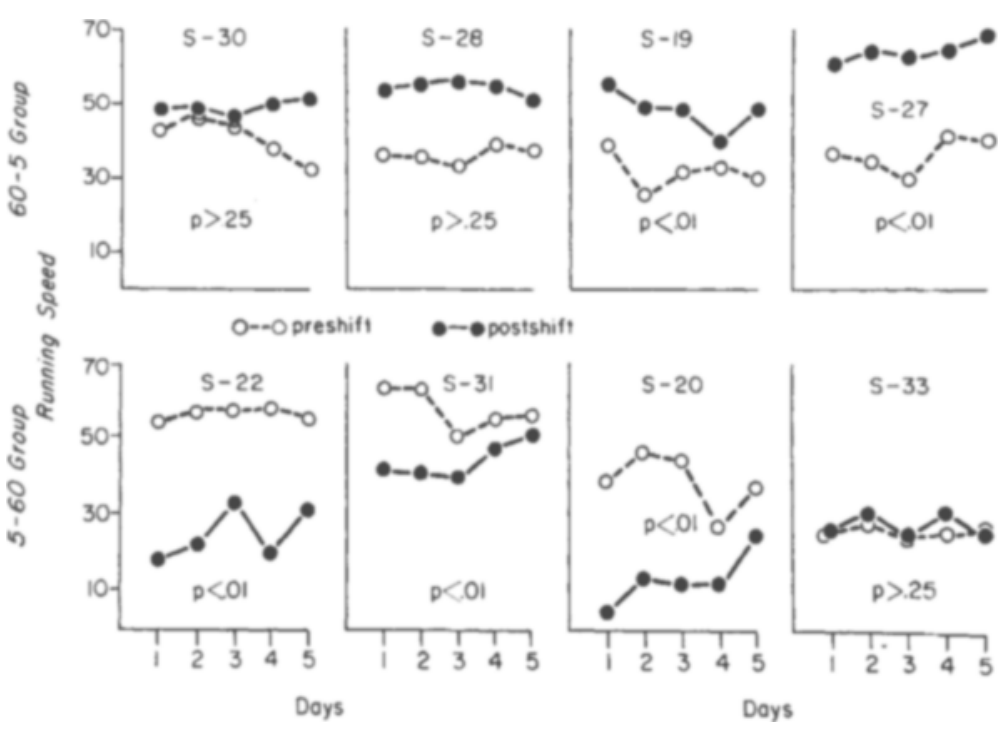

Fig. 2. Pre- and postshift day-by-day data for each $S$. The $p$ values on each graph are for the comparison between the performances on the first and last days of postshift testing. 
is there sufficient evidence of regression to worry about its implications? There is no regression effect in our $60-5$ group (see Fig. 1). (Their strongest regression effect was in the 60-5 group.) Notice, also, that our $60-5$ group was faster on the first postshift trial than on subsequent trials. Panksepp et al attribute significance to the fact that their $60-5$ group was slower on this trial than on subsequent trials. Our $5-60$ group shows some regression, but it does not approach the preshift baseline. Seven of our eight $S$ s were significantly above (60-5 group) or below (5-60 group) the preshift baseline on the final day of postshift testing. (Our study had 100 postshift trials vs 75 in theirs) and the eighth $S$ showed no regression-it did not show the effect at all (see Fig. 2). In the Panksepp et al study, 5 of 10 (or 6 of 10 -it is difficult to classify their S 6) showed a significant tendency to regress (as gauged by comparing the last postshift day against the first postshift day). In our study 4 of 8 showed such a tendency. Thus, taking the two studies together, about half the Ss showed any significant tendency to regress, let alone a regression to the baseline. One would expect several Ss to show "regression" by chance alone, since in both studies, under all conditions (pre- and postshift), Ss showed significant bidirectional day-to-day changes in performance (see Fig. 2). Thus, there was a decent chance of observing a "regression" when one compared any one day against any other day. We are not sure what the appropriate statistical test would be here, but it seems clear that not only are the implications of the effect unclear, it is unclear that there is an effect.

Finally, it is curious that Panksepp et al should cite in support of their argument Scott's (1967) report that there was no overnight decrement in well trained $S$ s. Scott's data contradict their own. Using 15 trials per day, Scott found no warm-up effect on the 5th, 6th, and 7 th day of training. After at least 6 days of training (and apparently more), at 15 trials a day, Panksepp et al not only report a strong warm-up effect, they refer to it as an effect which is "often found in animals running for ESB." That Scott did not find a warm-up effect is readily explained by a drive (or arousal) decay hypothesis: He used a 15-min intertrial interval.

\section{REFERENCES}

DEUTSCH, J, A. Behavioral measurement of the neural refractory period and its application to intracranial self-stimulation. Journal of Comparative \& Physiological Psychology, 1964, 58, 1-9.

DEUTSCH, J. A., ADAMS, D. W., \& METZNER, R. J. Choice of intracranial stimulation as a function of delay between stimulation and strength of competing drive. Journal of Comparative \& Physiological Psy chology, 1964, $57,241-243$.
GALLISTEL, C. R. Intracranial stimulation and natural rewards: Differential effects of trial spacing. Psychonomic Science, 1967, 9, 163-168.

PANKSEPP, J., GANDELMAN, R., \& TROWILL, $J$. The effect of intertrial interval on running performance for ESB. Psychonomic Science, $1968,13,135-136$.

SCOTT, J. W. Brain stimulation reinforcement with distributed practice: Effects of electrode locus, previous experience and stimulus intensity. Journal of Comparative \& Phy siological Psychology, 1967, 63, 175-183. NOTE

1. Gallistel, C. R., Rolls, E., \& Greene, D. Neuronal function inferred by behavioral and electrophysiological measurement of the neural refractory period. Submitted to Science, 1969.

\section{Reply to Gallistel}

JAAK PANKSEPP, RONALD GANDELMAN, and JAY A. TROWILL, University of Massachusetts, Amherst, Mass. 01002

Gallistel's comment (see this issue) on our study, "The effect of intertrial interval on running performance for ESB" (Panksepp, Gandelman, \& Trowill, 1968), includes some provocative data and terminological comments about the interpretation of performance maintained by rewarding electrical stimulation of the brain (ESB). Gallistel originally reported (1967) that increasing intertrial interval (ITI) from 5 to $60 \mathrm{sec}$ increases nunning speed of waterdeprived rats responding for water but decreases running speed when animals are responding for ESB. Our replication of this procedure confirmed Gatlistel's results. However, a trial-by-trial analysis of our data revealed that many $S$ s showed a regression of running speeds toward the preshift baseline across postshift days. Thus, the first postshift trial emphasized by Gallistel may not have been representative of subsequent postshift trials. In fact, it was our contention that such an incomplete presentation of data could be misleading.

Gallistel's comment consists of several arguments and data which he interprets as supporting the "drive-decay" position of Deutsch \& Howarth (1963) and of Gallistel (1964). Since Gallistel has now provided session-by-session and trial-by-trial data which can be directly compared to our own data, we are in a position to reply to his comments.

Before dealing with the specific comments of Gallistel, attention should be drawn to the over- and underresponding during postshift trials as compared to preshift levels (Fig. 1 of Gallistel's article). It is tempting to suggest a similarity between these overreactions and the positive (elation) and negative (depression) contrast effects found with upshifts and downshifts of ESB current intensity (Panksepp \& Trowill, 1969). Although ITI shifts have not yet been clearly defined as incentive manipulations, we propose that the similarity of effects following such shifts warrants this consideration.

In our original article we felt reticent about proposing that ITI shifts are, in fact, incentive shifts. Now, having had the opportunity to also view Gallistel's data, the close similarity between his data and our own, especially in the 5- to 60-sec group, buttresses this supposition. If ITI is an incentive variable, then it would be reasonable to expect that the higher incentive (5-sec ITI) should show more rapid performance than the lower-incentive condition, as is so commonly found in varying the amount of reward. Viewing ITI as an incentive variable, shifts in ITI should lead to changes in performance to the appropriate incentive level after an initial and transitory contrast effect, rather than to the preshift baseline. Such a contrast and complete return to appropriate baseline is strikingly clear in the 5-60 group (Fig. 1Gallistel's data). Gallistel suggests that the regression of postshift performance may merely represent spurious session-to-session fluctuations. However, the regression, rather than fluctuating, shows systematic trends toward the preshift baseline. The regression is not minimal as Gallistel implies, especially in the 5-60 animals, six of nine of which, in the two experiments, showed significant regression to baseline. In all, $50 \%$ of the Ss showed significant regression--an impressive percentage considering that if no true effect is present in the data only one $S$ out of 20 should reach the .05 level by chance alone. Furthermore, in all cases except one (Gallistel's S-27) regression occurred in the direction predicted by incentive contrast. It thus seems unlikely that the regression is either trivial or a mere reflection of random fluctuations.

Gallistel suggests that the difference between his drive interpretation and our incentive-motivation interpretation may rest purely on terminological grounds. Since the phenomenological aspects of rewarding 